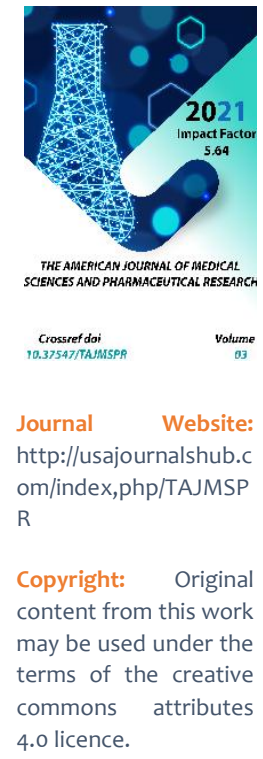

\title{
Microelemental Status In Chronic Kidney Disease
}

\author{
Razhabov Nodir Mukhammedovich \\ Bukhara State Medical Institute, Uzbekistan \\ Akhmedova Nilufar Sharipovna \\ Bukhara State Medical Institute, Uzbekistan
}

\section{ABSTRACT}

Evaluation of markers of endothelial dysfunction (endothelin-1 and nitric oxide) and elemental status (zinc, iron, magnesium) of chronic kidney disease (CKD). The study includes 104 patients with different stages of CKD: 63 patients with I stage of CKD, and 26 patients with CKD stage III and 15 patients with CKD V stage. Results: in patients with CKD, endothelin-1 increased with simultaneous decrease in production of nitric oxide in the progression of CKD from I to $V$ stage. The increase of the content of endothelin- 1 by 2.5 times determined in patients with stage $V$ CKD, which was accompanied by a decline of the ratio of the concentrations of NO/ET-1 by 5.5 times. In patients with CKD, elemental status impairments were detected, manifested by a decrease in the content of zinc, iron, and magnesium in the serum of blood at the first stage, with the maximum implementation in stage III CKD. Hypertension was detected in $68.3 \%$ of patients with CKD. Direct correlations were found between the content of serum iron and magnesium with the level of arterial pressure in patients with stage I CKD. In III and V stage CKD, a direct correlation of serum magnesium with blood pressure level and nitric oxide concentration was established. Conclusion: children with CKD have endothelial dysfunction and elemental status disorders, beginning with the first stage of CKD development, which can be considered as factors determining the progression of the pathological process in the kidneys.

\section{KEYWORDS}

Chronic kidney disease, endothelial dysfunction, blood pressure, zinc, iron, magnesium 


\section{INTRODUCTION}

Pathology of the urinary system occupies one of the leading places in the structure of morbidity. Chronic kidney disease accounts for $1 / 3$ of the nephrological pathology, while the incidence rate increases annually [1]. The concept of chronic kidney disease, reflecting the nature and rate of progression of pathology to end-stage renal failure, determines the need to search for markers of renal tissue damage as prognostically significant factors in the development of nephrosclerosis, which is of particular importance in childhood to optimize patient management [2]. In the development of renal pathology and especially in the occurrence of complications in chronic kidney disease, one of the main roles today is assigned to endothelial dysfunction. Endothelial dysfunction is a consequence of an imbalance between the production of vasoconstrictor, prothrombotic and proliferative compounds, on the one hand, and vasodilating, angioprotective, antiproliferative substances, on the other. At the same time, endothelial dysfunction is one of the leading links in the pathogenesis of kidney diseases of immune and non-immune genesis and an independent risk factor for the progression of chronic kidney disease [3].

Currently, the important role of macro- and microelements in the development of endothelial dysfunction has been established. According to the literature, magnesium is involved in the development of endothelial dysfunction by selectively reducing the release of nitric oxide by cells of the coronary endothelium; the latter serves as an endogenous vasodilator and inhibitor of platelet aggregation and adhesion [4, 5]. In addition, long-term magnesium deficiency in most cases is accompanied by an increased risk of cardiovascular pathology and may even lead to the death of the patient [4-6].

Zinc is part of the most important enzyme of the antioxidant system - superoxide dismutase.
Reduced activity of the antioxidant system leads to chronic oxidative stress and plays an important role in the pathogenesis of hypertension [6-8].

Iron, a metal with variable valence, readily promotes the formation of reactive oxygen species in the body, which can damage the endothelium. Iron deficiency in the body is considered one of the leading factors in the development of anemia in renal failure, which is accompanied by oxidative stress. Endothelial dysfunction, in turn, causes the syndrome of inadequate production of erythropoietin for hypoxia, aggravates the course of anemia and is a predictor of a higher risk of developing cardiovascular diseases [9].

Thus, a comprehensive study of markers of endothelial dysfunction together with homeostasis of zinc, iron and magnesium in children and adolescents with different stages of chronic kidney disease will determine their clinical significance in the progression of renal pathology.

\section{OBJECTIVE}

To assess markers of endothelial dysfunction (endothelin-1, nitric oxide) and elemental status in children at different stages of chronic kidney disease.

\section{MATERIAL AND METHODS}

Randomized cross-sectional study was carried out, which included 104 patients of chronic kidney disease. All patients were divided into three groups depending on the stage of the disease in accordance with the Clinical Guidelines for Chronic Kidney Disease of the US National Kidney Foundation (2002) and the Russian National Guidelines for Chronic Kidney Disease (2012). Group 1 consisted of 63 patients with stage I of the disease (GFR * greater than or equal to $90 \mathrm{ml} / \mathrm{min} / 1.73 \mathrm{mz}$ ), group $2-26$ patients with chronic stage III kidney disease 
(GFR from 59 to $30 \mathrm{ml} / \mathrm{min} / 1.73 \mathrm{mz}$ ), in group 3 - 15 patients with stage $V$ chronic kidney disease (GFR less than $15 \mathrm{ml} / \mathrm{min} / 1.73 \mathrm{mz}$ ). The control group consisted of 38 apparently healthy children matched by sex and age. All patients and / or their parents signed voluntary informed consent to participate in the study. The work was carried out on the basis of the nephrology department of the Children's Republican Clinical Hospital of the Ministry of Health of the Republic of Tatarstan. The study was approved by the local ethics committee of Kazan State Medical University.

The content of endothelin-1 in the blood serum was determined by the ELISA method using kits from Biomedica (Austria). The content of nitric oxide in the blood serum was judged by the level of its stable metabolites nitrites ( $\mathrm{NO}_{2}$ -) and nitrates ( $\mathrm{NO}_{3}-$ ), which were determined by the method of V.A. Metelskoy and N.G. Humanova (2005). The content of zinc, iron and magnesium in the blood serum was detected by atomic absorption spectrophotometry.

The research materials were subjected to statistical processing using the methods of parametric and nonparametric analysis in accordance with the results of checking the compared populations for normality of distribution. The accumulation, correction, systematization of the initial information and visualization of the results were carried out in Microsoft Office Excel 2016 spreadsheets. Statistical analysis was carried out using the IBM SPSS Statistics 23 program.

\section{RESULTS AND DISCUSSION}

The results of the study showed a significant increase in the concentration of endothelin- 1 in all studied groups compared with the control group ( $p<0.001)$. The production of nitric oxide was the highest in patients with stage I of chronic kidney disease and significantly exceeded the values of the control group ( $p$ $<0.001)$.

The study of the content of markers of endothelial dysfunction in the blood serum depending on the stage of chronic kidney disease using the Kruskal-Wallis test revealed a general trend for all studied parameters: the progression of the stage of chronic kidney disease was accompanied by a statistically significant increase in the level of endothelin-1 and a decrease in the content of nitric oxide in the blood serum ( $p<0.001$ in all cases). Comparison of the ratio of nitric oxide / endothelin-1 in patients and in the control group showed no changes in this parameter in children with stage I chronic kidney disease. As the disease progressed, there was a decrease in the median ratio of nitric oxide / endothelin1 from 55.63 conv. units with chronic kidney disease stage I up to 13.56 conv. units with chronic kidney disease stage $\mathrm{V}$.

Thus, the revealed increased concentration of endothelin-1 at stage I of chronic kidney disease may indicate a violation of the vasomotor function of the endothelium already in the early stages of the disease. With the progression of the disease, an increase in the level of endothelin-1 in the blood serum was noted, which demonstrates the great prognostic value of vasoconstriction at the stages of nephrosclerosis formation [10]. The maximum level of nitric oxide in children, identified by us at stage I of chronic kidney disease, promotes inhibition of endothelial dysfunction caused by hyperproduction of endothelin-1, which in these conditions is a favorable factor. As chronic kidney disease progressed in children, nitric oxide production significantly decreased, which was accompanied by a significant decrease in the nitric oxide / endothelin-1 ratio, the prevalence of vasopressor endothelin-1 levels, and the formation of endothelial dysfunction [10]. 
Considering that arterial hypertension is a nonimmune factor in the progression of renal pathology, all patients over five years of age with chronic kidney disease underwent 24-hour blood pressure monitoring, according to the results of which arterial hypertension was detected in $68.3 \%$ of patients, despite the ongoing antihypertensive therapy.

The one-way analysis of variance made it possible to establish statistically significant differences in blood pressure indicators depending on the stage of chronic kidney disease ( $p<0.05$ in all groups). In all cases, the mean values of the indicators increased with the progression of the disease. Nighttime blood pressure readings also had a general trend: statistically significant differences were observed when comparing the parameters of patients with stage $\mathrm{V}$ and I chronic kidney disease (for systolic blood pressure (SBP) - $p$ $<0.001$, for diastolic blood pressure (DBP) - $p$ $<0.001)$, when comparing the indicators of patients with chronic kidney disease stage $V$ and III (for both SBP and DBP - p = 0.01). A significant increase in nighttime blood pressure indicators was noted with the progression of the stage of chronic kidney disease.

The study of the elemental status in patients revealed a statistically significant decrease in the serum levels of zinc, iron and magnesium compared to the control group ( $p<0.001$ ). According to the results of the analysis of variance, the content of micro- and macroelements in the blood serum did not have significant differences depending on the stage of chronic kidney disease ( $p>0.05$ in all cases). However, it is necessary to note a general tendency towards a decrease in the content of zinc, iron and magnesium in the blood serum in patients with stage III chronic kidney disease and a tendency towards an increase in indicators in chronic kidney disease stage $V$.
We carried out a correlation analysis of the relationship between endothelial dysfunction indicators, the content of elements in the blood serum and blood pressure indicators in patients with chronic kidney disease. For patients with stage I of the disease, statistically significant inverse correlations of SBP and DBP with the iron content in the blood serum ( $p=$ 0.032 and $p=0.029$, respectively) and with the serum magnesium content $(p=0.043$ and $p=$ 0.047 , respectively) should be noted. We also assessed the prognostic significance of the content of iron and magnesium in the blood serum in order to determine the likelihood of an increase in daily SBP values above $130 \mathrm{~mm}$ $\mathrm{Hg}$. using the ROCcurve method. The critical level of serum iron was $1.013 \mu \mathrm{g} / \mathrm{ml}$, and magnesium was $1.835 \mathrm{mg} / \mathrm{dl}$. With a decrease in the content of these elements below the critical level, an increase in SBP above $130 \mathrm{~mm}$ $\mathrm{Hg}$ was predicted. Among patients with stages III and V of chronic kidney disease, a statistically significant inverse correlation of magnesium in serum with blood pressure indicators was also established: daytime SBP and nocturnal SBP ( $p$ $<0.001)$ and nocturnal DBP $(p=0.033)$ and $a$ direct correlation of magnesium content in serum with serum nitric oxide levels ( $r s=0.497$; $\mathrm{p}=0.046$ and $\mathrm{rs}=0.673 ; \mathrm{p}=0.013$, respectively).

\section{CONCLUSION}

Thus, the studies carried out have shown that endothelial dysfunction is observed in children with chronic kidney disease starting from stage I of the development of the disease. At the same time, the maximum increase (2.5 times) in the content of such a powerful vasoconstrictor as endothelin-1 was found in patients with stage $V$ chronic kidney disease, which was accompanied by a decrease in the concentration of the vasodilator of nitric oxide and a 5.5 -fold decrease in the ratio of nitric oxide / endothelin-1 in blood serum compared with the control group. This indicates the most 
pronounced changes in the functional state of the endothelium in patients with stage $V$ chronic kidney disease. Violations of the elemental status, manifested by a statistically significant decrease in the content of zinc, iron, magnesium in the blood serum, were detected already at stage I, with maximum realization at stage III of chronic kidney disease. Two elements were of diagnostic value for predicting daytime and nighttime SBP: magnesium and iron. With a decrease in iron content below $1.013 \mu \mathrm{g} / \mathrm{ml}$, and magnesium below $1.835 \mathrm{mg} / \mathrm{dL}$, an increase in SBP above $130 \mathrm{~mm} \mathrm{Hg}$ was predicted. The established statistically significant direct correlation of the serum magnesium content with the level of nitric oxide and inverse correlation with the blood pressure level allows us to conclude the participation of magnesium in the synthesis of nitric oxide and in the regulation of blood pressure in chronic kidney disease in children.

\section{REFERENCES}

1. Ivanova I.E. Chronic kidney disease in children and adolescents. Zdravoohranenie CHuvashii 2013; 2: 38-45. (in Russ)

2. Komarova O.V., Smirnov I.E., Kucherenko A.G., Tsygin A.N., Kostyushina I.S. Endothelial dysfunction in children with chronic kidney disease. Ros pediatr zhurn 2012; 5: 23-26. (in Russ)

3. Mel'nikova Yu.S., Makarova T.P. Endothelial dysfunction as the key link of chronic diseases pathogenesis. Kazanskij medicinskij zhurnal 2015; 96(4): 659-665. (in Russ)

4. Watson R.R., Preedy V.R., Zibadi S. Magnesium in human health and disease. New York: Humana Press, 2013; 309.

5. Kanbay M., Yilmaz M.I., Apetrii M., Saglam M., Yaman H., Unal H.U. et al. Relationship between serum magnesium levels and cardiovascular events in chronic kidney disease patients. Am J Nephrol 2012; 36(3): 228-237. DOI: $10.1159 / 000341868$
6. Habibrahmanova Z.R., Makarova T.P., Sadykova D.I. Metabolism of quantity elements and essential trace elements in patients with essential arterial hypertension in different ecological settings. Kazanskij medicinskij zhurnal 2013; 94(6): 798-803. (in Russ)

7. Maret W. Zinc biochemistry: from a single zinc enzyme to a key element of life. Adv Nutr 2013; 4(1): 82-91. DOI: 10.3945/an.112.003038

8. Lobo J.C., Stockler-Pinto M.B., Farage N.E., Faulin Tdo E., Abdalla D.S., Torres J.P. et al. Reduced plasma zinc levels, lipid peroxidation, and inflammation biomarkers levels in hemodialysis patients: implications to cardiovascular mortality. Renal Failure 2013; 35(5): 680-685. DOI: 10.3109/0886022X.2013.789960

9. Petuhov V. S., Zan'ko S.N. Iron deficiency and endothelial dysfunction as risk factors and diagnostic markers of placental insufficiency. Vestnik VGMU 2011; 10(3): 5564. (in Russ)

10. Smirnov I. E., Kucherenko A.G., Komarova O.V. Biomarkers of the formation of nephrosclerosis in chronic kidney disease in children. Ros pediatr zhurn 2014; 17(6): 1015. (in Russ) 